\title{
ESTEROLES PRESENTES EN EL EXTRACTO APOLAR DE LAS RAÍCES DE AJO SACHA Mansoa alliacea
}

\author{
Jenny E. Grovas Llamocca ${ }^{\mathrm{a}}$, Elena A. Cóndor Cuyubamba ${ }^{\mathrm{a}}$, \\ Ingrit E. Collantes Díaz ${ }^{b}$ Víctor M. Reyna Pinedo ${ }^{a^{*}}$
}

\begin{abstract}
RESUMEN
A partir del extracto apolar de las raíces de Ajo sacha Mansoa alliacea colectadas en el Jardín Botánico del Centro de Rehabilitación de Adicciones y de Investigación de Medicinas Tradicionales - Takiwasi (Tarapoto, región San Martín, en la Amazonía peruana) se identificaron el estigmasterol $(90,36 \%)$ y el $\gamma$ - sitosterol $(8,76 \%)$ como sus principales esteroles, los cuales fueron analizados mediante cromatografía de gases e identificados mediante sus espectros de masas. Adicionalmente, se realizó el análisis cualitativo de sus metabolitos secundarios.
\end{abstract}

Palabras clave: ajo sacha, Mansoa alliacea, estigmasterol, $\gamma$ - sitosterol, esteroles.

\section{STEROLS PRESENT IN THE APOLAR EXTRACT OF GARLIC ROOTS OF GARLIC SACHA Mansoa alliacea}

\begin{abstract}
From the apolar extract of the roots of garlic Sacha Mansoa alliacea collected in the Botanical garden of Center of Rehabilitation of addictions and research of traditional medicines - Takiwasi (Tarapoto, San Martin region, in the Peruvian Amazon) identified stigmasterol $(90,36 \%)$ and $\gamma$-sitosterol $(8,76 \%)$ as their main sterols, wich were analyzed by gas chromatography and identified by their mass spectra. In addition, was the qualitative analisys of the secondary metabolites.
\end{abstract}

Key words: Garlic sacha, Mansoa alliacea, stigmasterol, $\gamma$ - sitosterol, sterols.

\footnotetext{
a Facultad de Ciencias, Universidad Nacional de Ingeniería, Av. Túpac Amaru 210, Lima 25 - Perú,

*vrey26@yahoo.es

b Facultad de Ingeniería Química y Textil, Universidad Nacional de Ingeniería.
} 


\section{INTRODUCCIÓN}

El Perú es uno de los doce países con mayor diversidad biológica del mundo, conocidos como países megadiversos, tanto por el número de especies y de recursos genéticos como por la variedad de ecosistemas. Se calcula que posee 25 mil especies de plantas conocidas, de las cuales 5354 son endémicas de nuestro país ${ }^{1}$.

Esta riqueza vegetal permitió el desarrollo de la medicina tradicional y el uso de plantas medicinales en la práctica médica de las diversas culturas prehispánicas de nuestro país, en sus tres regiones, Costa, Sierra y Amazonía, uso tradicional que continúa hasta nuestros días.

El "ajo sacha", Mansoa alliacea (sinónimos: Adenocalymma alliaceum y Pseudocalymmma alliaceum $)^{1}$ es una especie que se encuentra, sobre todo, en los bosques primarios de zonas tropicales. Comparte su hábitat con especies como el aguaje, algodón, chuchuwasi, uña de gato, uvilla. En el Perú se encuentra, principalmente, en zonas de la selva alta y baja en las regiones de Amazonas, Huánuco, Loreto y San Martín².

Es una planta muy utilizada con fines medicinales por los grupos étnicos de la Amazonía peruana. Sus hojas, raíces y cortezas son utilizadas en diferentes preparaciones para tratar una variedad de dolencias. Su principal uso es como antirreumático, para tratar dolores e inflamaciones articulares y musculares en general. También se la utiliza para bajar la fiebre y para los dolores de cabeza ${ }^{2}$.

Las raíces y cortezas son preparadas en maceración alcohólica para tratar internamente el reumatismo y la artritis. Las hojas son empleadas en infusión como analgésico general o en emplastos para tratar externamente dolores en general (muscular, articular, de cabeza). Como planta maestra y tónico reconstituyente, se prepara la maceración acuosa de la raíz ${ }^{2}$.

En el Centro Takiwasi preparan la tintura de ajo sacha por un proceso de maceración de los tallos molidos en una mezcla de alcohol y agua (producto que es comercializado en todo el Perú).

En la bibliografía hemos encontrado un estudio fitoquímico relativo a las raíces de ajo sacha $\left(M\right.$. alliacea $^{3}$. Las otras publicaciones están referidas a los constituyentes presentes en las partes aéreas de esta planta (hojas y tallos) ${ }^{4-13}$ (tabla 1). 
Tabla 1. Estudios químicos publicados acerca de ajo sacha (Mansoa alliacea)

\begin{tabular}{|c|c|c|c|c|}
\hline $\mathrm{N}^{0}$ & $\begin{array}{c}\text { Parte } \\
\text { vegetal }\end{array}$ & $\begin{array}{l}\text { Procedencia } \\
\text { de la planta }\end{array}$ & Constituyentes químicos & Referencia \\
\hline 1 & $\begin{array}{l}\text { Raíces y } \\
\text { Hojas }\end{array}$ & India & $\begin{array}{l}\text { Análisis cualitativo; } \\
\text { análisis cuantitativo (fenoles) }\end{array}$ & $\begin{array}{l}\text { Patel et al. } \\
(2013)^{3}\end{array}$ \\
\hline 2 & Hojas & Brasil & $\begin{array}{l}\text { Compuestos fenólicos (luteolina, } \\
\text { Ac. p- cumarina, Ac.fenólico, etc.) }\end{array}$ & $\begin{array}{l}\text { Faccin et al. } \\
\qquad(2017)^{4}\end{array}$ \\
\hline 3 & Hojas & Brasil & $\begin{array}{l}\text { Compuestos fenólicos (Ac. p- } \\
\text { Cumarina, Ac. fenólico) }\end{array}$ & $\begin{array}{l}\text { Da Silveira et al. } \\
\qquad(2015)^{5}\end{array}$ \\
\hline 4 & Hojas & Brasil & $\begin{array}{l}\text { Triterpenoides } \\
\text { (ácido betulínico) }\end{array}$ & $\begin{array}{l}\text { Gobo et al. } \\
(2016)^{6}\end{array}$ \\
\hline 5 & Tallos & $\begin{array}{l}\text { Perú- } \\
\text { Iquitos }\end{array}$ & Naftoquinonas & $\begin{array}{l}\text { Itokawaq et al. } \\
\qquad(1992)^{7}\end{array}$ \\
\hline 6 & Hojas & Brasil & $\begin{array}{l}\text { Triterpenos (Ac. ursólico } \\
\text { y Ac. Oleanólico) }\end{array}$ & $\begin{array}{l}\text { Trindade et al. } \\
\qquad(2007)^{8}\end{array}$ \\
\hline 7 & Hojas & $\begin{array}{l}\text { Perú-Madre } \\
\text { de Dios }\end{array}$ & $\begin{array}{l}\text { Compuestos organosulfurados } \\
\text { (alil trisulfito, dialil disulfito) }\end{array}$ & $\begin{array}{l}\text { Olivera et al. } \\
\quad(2013)^{9}\end{array}$ \\
\hline 8 & $\begin{array}{l}\text { Tallos y } \\
\text { corteza }\end{array}$ & Perú & $\begin{array}{l}\text { Quinonas } \\
\text { ( } \alpha \text {-lapachonas) }\end{array}$ & $\begin{array}{l}\text { Lock }(2016)^{\mathbf{1 0}} \\
\text { Alvarez et al. } \\
(1993)^{\mathbf{1 1}}\end{array}$ \\
\hline 9 & Hojas & India & Triterpenos pentacíclicos & $\begin{array}{l}\text { Misra et al. } \\
(1995)^{\mathbf{1 2}}\end{array}$ \\
\hline 10 & Hojas & India & $\begin{array}{l}\text { Compuesto órgano sulfurado } \\
\text { (alliina) }\end{array}$ & $\begin{array}{l}\text { Apparao et al. } \\
(1981)^{\mathbf{1 3}}\end{array}$ \\
\hline
\end{tabular}

Al respecto, es pertinente tener presente que la composición química de una planta puede variar mucho según la parte de la planta que estamos tratando: las flores tienen diferentes constituyentes que las hojas, y estas pueden ser muy diferentes que las presentes en las raíces o tallos. Asimismo, la composición química de la planta varía mucho en función de varios factores, tales como las condiciones de siembra de la planta (tipo de suelo, altitud, humedad y temperatura ambiental), la edad de la planta, la época y hora de colecta, etc. ${ }^{14-15}$. Igualmente, según el lugar geográfico, una especie vegetal cultivada en la India puede tener una composición muy diferente a la misma planta (especie) cultivada en la Amazonía peruana.

Los esteroles (fitosteroles) son alcoholes esteroidales constituidos por un anillo tetracíclico del ciclopentano $[\alpha]$ fenantreno con una larga cadena lateral flexible, enlazada al átomo de carbono 17 (figura 1). Los cuatro anillos, denominados A, B, C y D, de izquierda a derecha, tienen enlaces de anillo trans, formando un sistema plano $\alpha$. Los más representativos son el $\beta$-sitosterol, el estigmasterol y el campesterol. El $\gamma$-sitosterol es un $24 \beta$-epímero del $\beta$-sitosterol. Los sitosteroles se diferencian del estigmasterol solo en que la cadena lateral del sitosterol es saturada16. El $\beta$-sitosterol es el principal esterol presente en las plantas, mientras que muy poco se ha publicado acerca del $\gamma$-sitosterol ${ }^{16}$. 


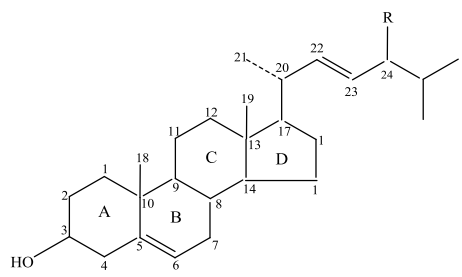

Estructura básica de los esteroles

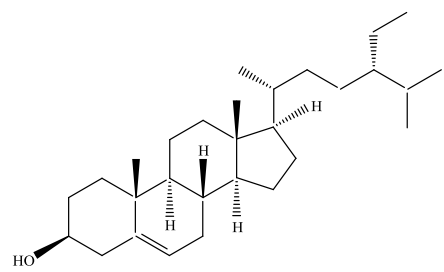

$\gamma$ - sitosterol

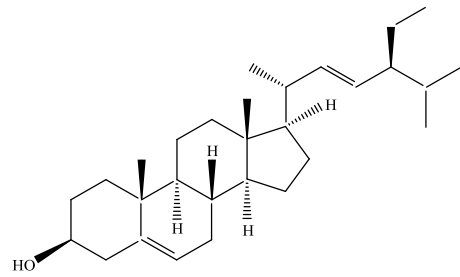

Estigmasterol

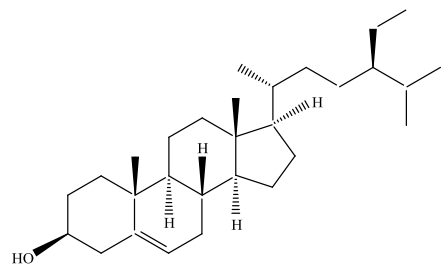

$\beta$ - sitosterol

Figura 1. Estructura de los principales fitosteroles

\section{Propiedades farmacológicas de los esteroles}

Debido a su mayor presencia en las plantas, se han estudiado más las actividades farmacológicas del $\beta$-sitosterol y del estigmasterol17, pero poco se ha reportado acerca del $\gamma$-sitosterol16. El estigmasterol inhibe marcadamente los tumores en carcinogénesis en ratas y presenta efecto inhibitorio sobre la transcriptasa reversa del VIH17; además, se ha verificado que posee potenciales propiedades antiinflamatorias 18. Extractos vegetales que contienen de 10-20\% de $\gamma$-sitosterol exhiben un amplio rango de actividad citotóxica frente a células cancerosas 19.

A continuación se describe el estudio químico cualitativo y el análisis mediante cromatografía de gases acoplada con espectrómetro de masas (CG-EM) que permitió la identificación de dos esteroles presentes en las raíces de "ajo sacha" (Mansoa alliacea), estigmasterol y $\gamma$-sitosterol.

\section{PARTE EXPERIMENTAL}

\section{Materiales y equipos}

Para las cromatografías en capa fina (CCF) se utilizaron placas de sílica Kieselgel 60 F254,0,2 mm (Riedel de Haën) y las cromatografías en columna (CC*) se realizaron sobre sílica gel.

\footnotetext{
${ }^{*}$ CC: Sigma-Aldrich, tamaño de poro $60 \AA$ A, tamaño de partícula 35-75 $\mu \mathrm{m}$.

Una muestra botánica de esta planta fue colectada en dicho Centro en el mes de febrero de 2014 e identificada por la Dra. Haydeé Montoya T., Jefa del Herbario San Marcos del Museo de Historia Natural de la Universidad Nacional Mayor de San Marcos (05.May.2014).
} 
El análisis e identificación de esteroles se realizó en un cromatógrafo de gases acoplado a masas (CG-EM), cromatógrafo SHIMADZU, GC-2010 Plus, con automuestreador: AOC6000, y detector de espectrometría de masas SHIMADZU, GCMS-QP210 Ultra. Columna GC: RESTEK. RTX-5MS, 30m x 0.25 mm ID x $0.25 \mu \mathrm{m}$ df. Serial: 1346249.

\section{Material vegetal}

Las raíces de ajo sacha (Mansoa alliacea) se colectaron en el Jardín Botánico del Centro de Rehabilitación de Adicciones y de Investigación de Medicinas Tradicionales - Takiwasi, en el mes de febrero de 2017 por el Dr. Fernando Mendive, Director Científico.

La muestra fue cortada en trozos pequeños y se secó a 400C por 70 días. Se pulverizó a grano semifino en un molino doméstico.

\section{Análisis cualitativo}

El análisis cualitativo (marcha fitoquímica) se realizó por duplicado según el método descrito por Rondina \& Coussio ${ }^{20}$.

\section{Extracción, separación e identificación de esteroles}

\section{Extracción}

Las raíces secas y molidas $(196,15 \mathrm{~g})$ se maceraron en éter de petróleo (1,3 L), a temperatura ambiente durante 24 horas. El extracto orgánico (aprox. 500mL) se retiró con ayuda de una pipeta volumétrica de $50 \mathrm{~mL}$. Esta extracción por maceración a temperatura ambiente se repitió tres veces adicionales, durante ocho, dos y cinco días, respectivamente. Se juntaron los extractos orgánicos (aprox. 2,8 L) y el solvente se eliminó a 400C y presión reducida (rotavapor), obteniéndose $643 \mathrm{mg}$ de extracto bruto orgánico (EBO), sólido de color pardo claro.

\section{Separación por cromatografía en columna (CC)}

El EBO $(643 \mathrm{mg})$ fue fraccionado mediante cromatografía en columna sobre sílica Gel*, utilizando como eluente, primero, una mezcla de acetato de etilo-EtOAc en éter de petróleo (10\% y $20 \%$, respectivamente), luego, acetato de etilo en cloroformo-CHCl3 (50\%) y finalmente metanol-MeOH, obteniéndose en total 52 fracciones (de $10 \mathrm{~mL} \mathrm{c} / \mathrm{u}$ ), las cuales, luego de ser analizadas mediante cromatografía en capa fina $(\mathrm{CCF})^{* *}$, fueron reunidas en ocho grupos (extractos G1 a G8).

Al evaporar el solvente del extracto $\mathrm{G} 4(70 \mathrm{~mL})$, mediante destilación a vacío (rotavapor), se observó la formación de cristales blancos impuros $(55,5 \mathrm{mg})$, los cuales fueron parcialmente purificados mediante recristalización con éter de petróleo, obteniéndose 23,4 mg de un sólido blanco amarillento (al que continuamos denominando extracto G4).

\footnotetext{
* Sílica gel de alta pureza, de tamaño de poro $60 \AA$ de 220-440 mesh.

** CCF: Placas de Kieselgel 60 F254, 0,2 mm (Riedel de Haën), eluente: éter: acetato de etilo (9:1), revelador: H2SO4 al $50 \%$.
} 


\section{Análisis del extracto G4 por CG-EM}

La inyección de la muestra se realizó por el modo "Split" con una relación 1:10, siendo la temperatura del inyector, la interfase y el detector, $2800 \mathrm{C}, 2500 \mathrm{C}$, y $2000 \mathrm{C}$, respectivamente. El volumen de inyección empleado fue $0,5 \mu \mathrm{l}$ de la muestra preparada $\left(1,2 \mathrm{mg} / \mathrm{mL} \mathrm{CH}_{2} \mathrm{Cl}_{2}\right)$.

La separación cromatográfica se realizó en una columna Rtx-5MS (Crossbond $5 \%$, difenil $95 \%$, dimetil polisilano) ( $30 \mathrm{~m} \times 0,25 \mathrm{mmx} 0,25 \mu \mathrm{m})$ y como gas portador se utilizó He 99,99 $\%$ de pureza, con un flujo de $0,8 \mathrm{~mL} / \mathrm{ml}$. La temperatura del horno se programó a $2400 \mathrm{C}$, con aumento de temperatura de una velocidad de $100 \mathrm{C} / \mathrm{min}$. hasta $2650 \mathrm{C}$, temperatura que permaneció durante 40 minutos. El detector operó el modo de ionización por impacto electrónico (IE) $(70 \mathrm{eV})$ a 2000C. La detección se realizó en el modo de barrido desde 35 a $500 \mathrm{~m} / \mathrm{z}$.

\section{Identificación de esteroles por EM}

La identificación de los compuestos analizados mediante CG pudo realizarse comparando sus espectros de masas (patrones de fragmentación) con aquellos presentes en la base de espectros de masas NIST-2014, incorporado en el equipo CG-EM.

Además, se compararon los espectros de masas obtenidos con aquellos publicados en la bibliografía.

\section{RESULTADOS Y DISCUSIÓN}

\section{Análisis cualitativo}

El análisis cualitativo mostró que las raíces de ajo sacha (Mansoa alliacea) contiene aminogrupos primarios o secundarios, grupos fenólicos libres, triterpenos y esteroides, quinonas, antronas o antranoles, alcaloides y catequinas.

\section{Análisis e identificación de esteroles por CG-EM}

La figura 2 muestra el cromatograma obtenido mediante cromatografía de gases del extracto G4.

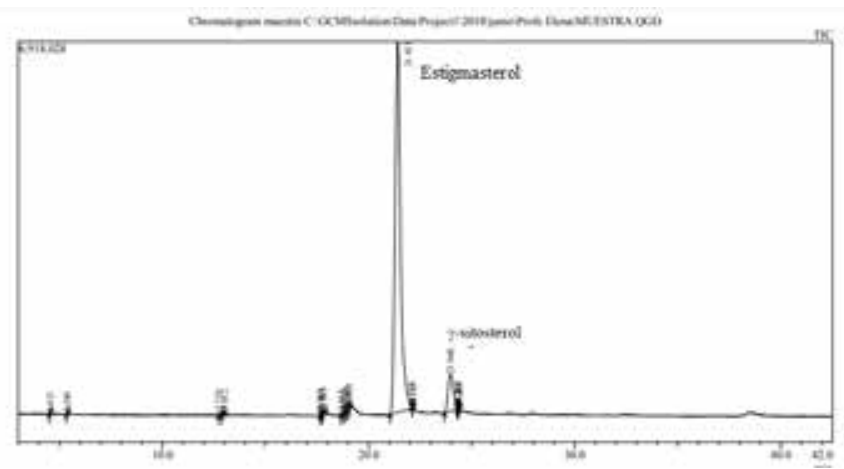

Figura 2. Cromatograma de iones totales obtenido a partir del extracto G4 de las raíces de ajo sacha (Mansoa alliacea) 
En la tabla 2 se presentan solo los dos compuestos mayoritarios del extracto apolar de las raíces del ajo sacha, los esteroles: estigmasterol $(90,36 \%)$ y $\gamma$-sitosterol $(8,76 \%$,). Los otros 16 constituyentes reportados por el equipo tienen concentraciones inferiores a 0,23\% (uno de ellos) y el resto porcentajes inferiores a $0,09 \%$.

Tabla 2. Compuestos identificados con el empleo de la cromatografía de gases acoplada a espectrometría de masas

\begin{tabular}{ccc}
\hline Compuesto & $\mathrm{T}_{\mathrm{R}}(\mathrm{min})$ & Area de Pico (\%) \\
\hline Estigmasterol & 21,423 & 90,36 \\
$\gamma$-sitosterol & 23,968 & 8,76 \\
\hline
\end{tabular}

El equipo CG-EM proporciona los espectros de masas que corresponde a cada compuesto analizado (según su tiempo de retención) y proporciona los espectros de masas de las sustancias de referencia que están registrados en la base de espectros del equipo, resultando positiva la identificación de ambos esteroles.

El espectro de masas del compuesto mayoritario (90,36 \%, figura 3) resulto similar al espectro del estigmasterol (registrado en la base de espectros del equipo), ambos con el pico ion molecular de $412 \mathrm{~m} / \mathrm{z}$.

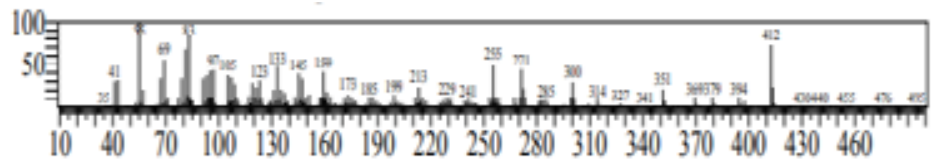

Figura 3. Espectro de masas del estigmasterol presente en el extracto apolar de las raíces del ajo sacha (Mansoa alliacea).

Además, este espectro de masas (figura 3) es similar al publicado por Mangas R. et al. (2008), en el análisis por CG-EM del extracto apolar de Clusia minor L. $^{17}$, e identificado como estigmasterol.

El espectro de masas del compuesto secundario (8,76 \%, figura 4) resulto similar al espectro del $\gamma$-sitosterol (registrado en la base de espectros del equipo), ambos con el pico ion molecular de $414 \mathrm{~m} / \mathrm{z}$.

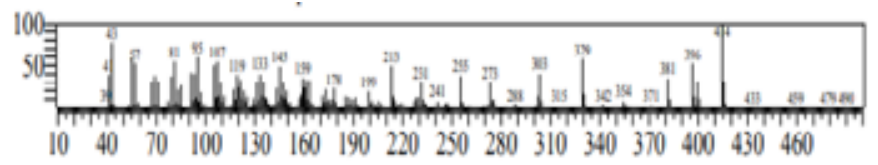

Figura 4. Espectro de masas del $\gamma$-sitosterol presente en las raíces del ajo sacha (Mansoa alliacea). 
Además, este espectro de masas (figura 4) es similar al publicado por Sheng \& Chen (2009) ${ }^{16}$, e identificado como $\gamma$-sitosterol.

\section{CONCLUSIONES}

A partir del extracto apolar de las raíces de ajo sacha, colectadas en el Jardín Botánico del Centro Takiwasi (Tarapoto, región San Martín, Amazonía peruana), se identificaron el estigmasterol $(90,36 \%)$ y el $\gamma$-sitosterol $(8,76 \%)$ como sus principales esteroles.

\section{AGRADECIMIENTO}

Se agradece al Vicerrectorado de Investigación de la Universidad Nacional de Ingeniería, por el apoyo económico brindado para la realización de este trabajo de investigación, al Dr. Fernando Mendive, Director Científico del Centro de Rehabilitación de Adicciones y de Investigación de Medicinas Tradicionales - Takiwasi por la muestra proporcionada. Al Mag. Pedro Baldera Aguayo por la información bibliográfica y la revisión del presente artículo.

\section{REFERENCIAS BIBLIOGRÁFICAS}

1. Brack A. Diccionario enciclopédico de plantas útiles de Perú. Cusco: Centro de Estudios Regionales Andinos "Bartolome de las Casas"; 1999.

2. Ajo sacha. [Internet] Laboratorio de Productos Naturales, Centro Takiwasi; 2013 [citado 13 abril 2018]. Disponible en: http://www.newlab.takiwasi.com/productos/AjoSachaEC. pdf

3. Patel I, Sipai S, Rathod D, Shrimali G, Patel A, Rami E. Phytochemical studies on Mansoa alliacea (Lam.). IJAPR. 2013; 4(6): 1823-1828.

4. Faccin H, Loose RF, Viana C, Lameira OA, Machado de Carvalho L. Determination of phenolic compounds in extracts of Amazonian medicinal plants by liquid chromatography-electrospray tándem mass spectrometry. Anal Methods. 2017; 9: 11411151.

5. Da Silveira GD, Jung Motta M, Sabo Muller L, Lameira O, Athayde ML, Piana M. et. al. Determination of Phenolic Antioxidants in Amazonian Medicinal Plants by HPLC with Pulse Amperometric Detection. J Liq Chromatogr Relat Technol. 2015; 38: 1259-1266.

6. Gobo LA, Viana C, Lameira OA, Machado de Carvalho L. A liquid chromatographyatmospheric pressure photoionization tandem mass spectrometric (LC-APPI-MS/ MS) method for the determination of triterpenoids in medicinal plant extracts. J Mass Spectrom. 2016; 51: 558-565.

7. Itokawa H, Matsumoto K, Morita H, Takeya K. Cytotoxic naphthoquinones from Mansoa alliacea. Phytochem. 1992; 31(3): 1061-1062. 
8. Trindade NS, Almeida ES, Arruda AC, Arruda MSP, da Silva MN, Santos LS, et al. Triterpenos Isolados das Folhas de Mansoa alliacea (Bignoniaceae). En: $30^{\circ}$ Reunião Anual da Sociedade Brasileira de Quimica, 31 mayo - 03 junio 2007; Águas de Lindóia, São Paulo, Brasil.

9. Olivera-Condori M, Flores-Arizaca J, Vasquez Zavaleta T, Ocsa-Borda E. Propiedades Fisicoquímicas y Bioactivas in Vitro del aceite esencial de Mansoa alliacea (LAM.) A. GENTRY. El CEPROSIMAD. 2013; 2(1): 96-102.

10. Lock O. Investigación Fitoquímica. Métodos en el estudio de Productos Naturales. Lima: Pontificia Universidad Católica del Perú; 2016.

11. Alvarez C, Hubiecki P, Lock O, Elin E. Quinonas de Pseudocalymma alliaceum. En: II Congreso Italo-Peruano de Etnomedicina, 1993; Lima, Perú.

12. Misra TN, Singh RS, Pandey HS, Prassad C. A novel pentacyclic triterpene acid from Adenocalymma Alliaceum leaves. J Nat Prod. 1995; 58(7): 1056-1058.

13. Apparao M, Kjer A, Olsen O, Venkata E, Rasmussen K, Sorensen H. Alliin in the garlicky taxon Adenocalymma alliaceum (Bignoniaceae). Phytochem. 1981; 20(4): 822-823.

14. Garg V, Dhar VJ, Sharma A, Dutt R. Facts about standardization of herbal medicine: a review. Zhong Xi Yi Jie He Xue Bao. 2012;10(10):1077-83.

15. Calixto J. Efficacy, safety, quality control, marketing and regulatory guidelines for herbal medicines (phytotherapeutic agents). Braz J Med Biol Res. 2000; 33(2): 177-189.

16. Sheng Y, Chen X-B. Isolation and identification of an isomer of $\beta$-sitosterol by HPLC and GC-MS. HEALTH. 2009; 1: 203-206.

17. Mangas Marin R, Montes de Oca Porto R, Bello Alarcon A, Nival Vasquez A. Caracterización por Cromatografía de gases/Espectrometría de Masas del Extracto Apolar de las Hojas de Clusia minor L. Lat Am J Pharm; 2008; 27(5): 747-751.

18. Gabay O, Sanchez C, Salvat C, Chevy F, Breton M, Nourissat G. et al. Stigmasterol: a phytosterol with potential anti-osteoarthritic properties. Osteoarthritis Cartilage. 2010; 18: 106-116.

19. Luo H, Cai Y, Peng Z, Liu T, Yangt S. Chemical composition and in vitro evaluation of the cytotoxic and antioxidant actiovities of supercritical carbon dioxide extracts of pitaya (dragon fruit) peel. Chem Cent J. 2014; 8: 1-7.

20. Rondina R, Coussio J. Estudio Fitoquímico de Plantas Medicinales. Rev Investig. Agropecu. 1969; 6 (33): 351-366. 